\title{
Relación del Foramen Lingual con Espinas Mentonianas y Evaluación del Conducto Lingual Mediante Radiografías Periapicales y Extraorales
}

\author{
Relationship Between Lingual Foramen and Mental Spines, and Evaluation \\ of Lingual Canal by Using Periapical and Extra-Oral Radiographs
}

Alejandro Hidalgo R. \& Jaime San Pedro V.

HIDALGO, A. \& SAN PEDRO, J. Relación del foramen lingual con espinas mentonianas y evaluación del conducto lingual mediante radiografías intra y extraorales. Int. J. Odontostomat., 4(3):295-302, 2010.

RESUMEN: La literatura tradicional odontológica describe la ubicación radiográfica del foramen lingual en la cara lingual de la mandíbula, de ubicación media entre las espinas mentonianas (apófisis geni) en radiografías periapicales y panorámicas. Tradicionalmente se señala que las espinas mentonianas son las responsables del área radiopaca que rodea al foramen lingual. La ubicación radiográfica en posición mediana del foramen lingual con respecto a las espinas mentonianas es objeto de controversia. El objetivo de la presente investigación fue determinar la relación del foramen lingual con las espinas mentonianas en observación directa de mandíbulas secas, y mediante radiografías periapicales y panorámicas de las mismas, determinar la trayectoria del conducto lingual en tomografías lineales de la zona, y describir su representación radiográfica desde la perspectiva absorso-proyeccional. Esta investigación demostró la no concordancia radiográfica entre las espinas mentonianas y el área radiopaca que rodea al foramen lingual.

PALABRAS CLAVE: radiografía dental, línea mediana mandibular, espinas mentonianas, foramen lingual, conducto lingual, radiografía panorámica.

\section{INTRODUCCIÓN}

Las características anatómicas de la línea media mandibular y sus variantes debieran ser tomadas en cuenta durante procedimientos quirúrgicos en esta región (Tepper et al., 2001; Liang et al., 2006). Esto debe ser considerado durante la interpretación radiográfica (Gibilisco, 1994; Baldissera \& Silveira, 2002) con el objeto de evitar posibles complicaciones intraoperatorias y post quirúrgicas (McDonnell et al., 1994; Nagar et al., 2001; Isaacson, 2004; Jacobs \& Lambrichts, 2006; Liang et al., 2007; Rosano et al., 2009).

La literatura radiológica tradicional odontológica describe al foramen lingual como un pequeño agujero ubicado en la cara lingual de la mandíbula, en la línea media, observable en radiografías periapicales y panorámicas como un pequeño círculo radiolúcido, rodeado de un área radiopaca que correspondería a las espinas mentonianas (Gibilisco; De Freitas et al., 2002; Langlais \& Kasle, 1992; Haring \& Lind, 1997), localizado en ra- diografías periapicales bastante por debajo de los ápices de los incisivos centrales, aproximadamente a un centímetro bajo la línea inter-apical de los incisivos inferiores, de tamaño y frecuencia variables, con su diámetro rara vez superior a 1-2 mm (Wood \& Goaz, 1999).

Algunos autores señalan una baja frecuencia de aparición del foramen lingual en radiografías periapicales (Basrani et al., 2002). Se han reportado frecuencias de un $70 \%$ en radiografías periapicales y oclusales, con porcentajes superiores en radiografías panorámicas (92\%) en comparación con intraorales (Mraiwa et al., 2003) y una tendencia a aumentar su frecuencia de aparición $(97,05 \%)$ al utilizarse la tomografía computarizada para su observación (Kwai, 2007). A la observación directa de mandíbulas secas se ha reportado una alta frecuencia de aparición: 72,5\% ( Nagar et al.) a 99,04\% ( McDonnell et al.). 
Con respecto al número de forámenes linguales, mayoritariamente es único (64\%-74\%) (Mraiwa et al.; Liang et al., 2006), menos frecuentemente se encuentra doble (22-34\%), y pocas veces son más de dos (35\%) (Vasconcellos et al., 2000; Liang et al., 2006).

En relación con la ubicación anatómica del foramen lingual, se ha descrito una posición inferior, ubicada bajo las eminencias mentonianas, y una posición superior, ubicada entre o por sobre las eminencias mentonianas (Mraiwa et al.; Liang et al., 2007), siendo la ubicación superior más frecuente $(62 \%)$ respecto de la ubicación inferior (38\%) (Liang et al., 2007). Se ha reportado que cuando se presenta un solo conducto, su ubicación típica es superior, mientras que en casos de dos o más foraminas, la segunda se ubica siempre en posición inferior a las espinas mentonianas (Mraiwa et al.; Liang et al., 2007).

En cuanto a la trayectoria del conducto lingual, ésta se describe mayoritariamente ascendente (de delante-atrás, abajo-arriba) (72\%); observándose en un $28 \%$ una trayectoria descendente (de delante-atrás, arriba-abajo) (Liang et al., 2007)(Fig.1). Además, se ha observado una trayectoria única en la mayoría de los casos $(98,75 \%)$, mientras que canales con bifurcación se observan en muy pocas oportunida$\operatorname{des}(1,25 \%)$.

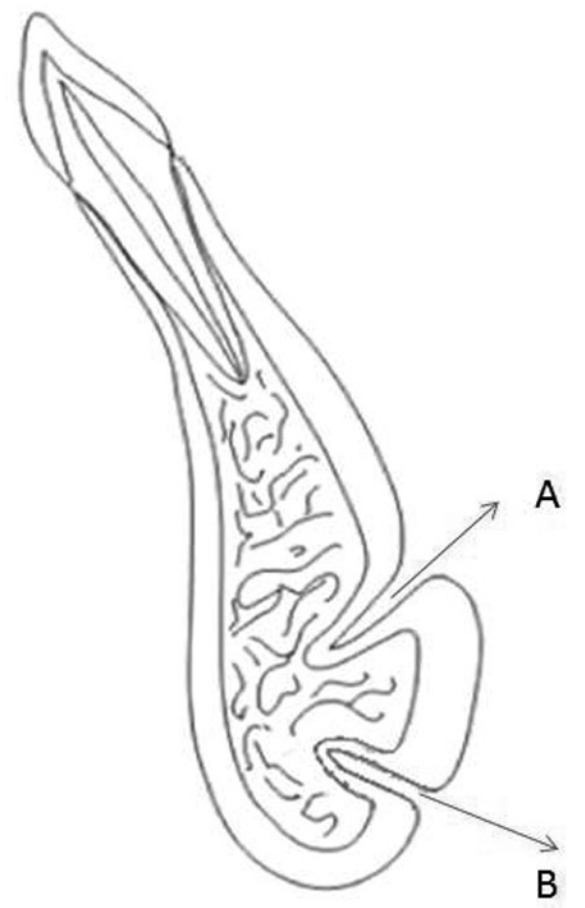

Fig. 1. Letra A indica trayectoria ascendente del conducto lingual. Letra B indica trayectoria descendente del conducto lingual.
La interpretación del área radiopaca alrededor del foramen lingual ha sido controversial, ya que se ha asociado tradicionalmente a la imagen de las espinas mentonianas, mientras que varios autores han reportado que dicha área no corresponde a éstas (McDonnell et al.; Goaz \& White, 1995; Baldissera \& Silveira; Mraiwa et al.; White \& Pharoa, 2004). Si bien hace bastante tiempo Benkow(1961) ya había señalado que las espinas mentonianas no generaban el área radiopaca en torno al formen lingual (Gomez Mattaldi, 1979; Baldissera \& Silveira), la descripción clásica se ha mantenido. Incluso se ha señalado que varios textos de anatomía no mencionan este reparo anatómico (McDonnell et al.; Baldissera \& Silveira; Liang et al., 2007), situación que fue constatada en la presente investigación (Velayos \& Santana, 1998; Rouvière \& Delmas, 1999; Figún \& Garino, 2002).

El objetivo de la presente investigación fue comparar la relación del foramen lingual con las espinas mentonianas por observación directa en mandíbulas secas, con relación a la observación mediante radiografías periapicales y panorámicas de las mismas, determinar la trayectoria del conducto lingual en tomografías lineales de la zona, y describir su representación radiográfica desde una perspectiva absorsoproyeccional.

\section{MATERIAL Y MÉTODO}

Se realizó un muestreo no probabilístico por conveniencia. Se analizaron nueve mandíbulas secas, pertenecientes al Departamento de Anatomía de la Facultad de Ciencias de la Salud de la Universidad de Talca. La edad y sexo de las muestras era desconocida.

Los criterios de inclusión fueron: (1) Mandíbulas en adecuada nivel de conservación que permitieran observar los reparos anatómicos y éstos se encontraran indemnes, (2) mandíbulas sin evidencia de fractura en línea media ni rasgos patológicos que sesgaran la observación. El criterio de exclusión fue la presencia de dimorfosis.

A esta muestra se les tomó radiografías periapicales de la zona de línea media sin marcadores en una primera etapa y con marcadores metálicos en los reparos anatómicos a estudiar: espinas mentonianas, foramen lingual, con un equipo Siemens Sirona $7 \mathrm{~mA}, 60 \mathrm{Kv}\left(-20^{\circ}, 0,25\right.$ segundos $)$. Posterior- 
mente a cada mandíbula de le tomó una radiografía panorámica (Programa 1,60Kv/9mA) sin marcadores, y una tomografía lineal de línea media (programa 23, $60 \mathrm{Kv}, 9 \mathrm{~mA}$ ) con marcadores en las espinas mentonianas y conducto lingual, con un equipo Orthophos Multiplus (Sirona Dental Systems, Germany). Se utilizaron láminas de estaño plomado a manera de filtro, ubicadas a la salida del tubo de rayos, y láminas de cobre adosadas a la línea media de la cara lingual de cada mandíbula para la radiografía panorámica para obtener una densidad adecuada en la zona de interés. Las radiografías y mandíbulas fueron evaluadas por un radiólogo maxilofacial.

\section{RESULTADOS}

A la observación directa del foramen lingual, sólo en dos mandíbulas se encontró entre las espinas mentonianas, sin embargo ambos casos en las radiografías periapicales no fueron las causantes del área radiopaca periférica al foramen lingual (Fig.2). En las siete mandíbulas restantes, los forámenes linguales no estuvieron relacionados con las espinas mentonianas, ni durante la observación directa de las mandíbulas, ni en la observación de las radiografías.

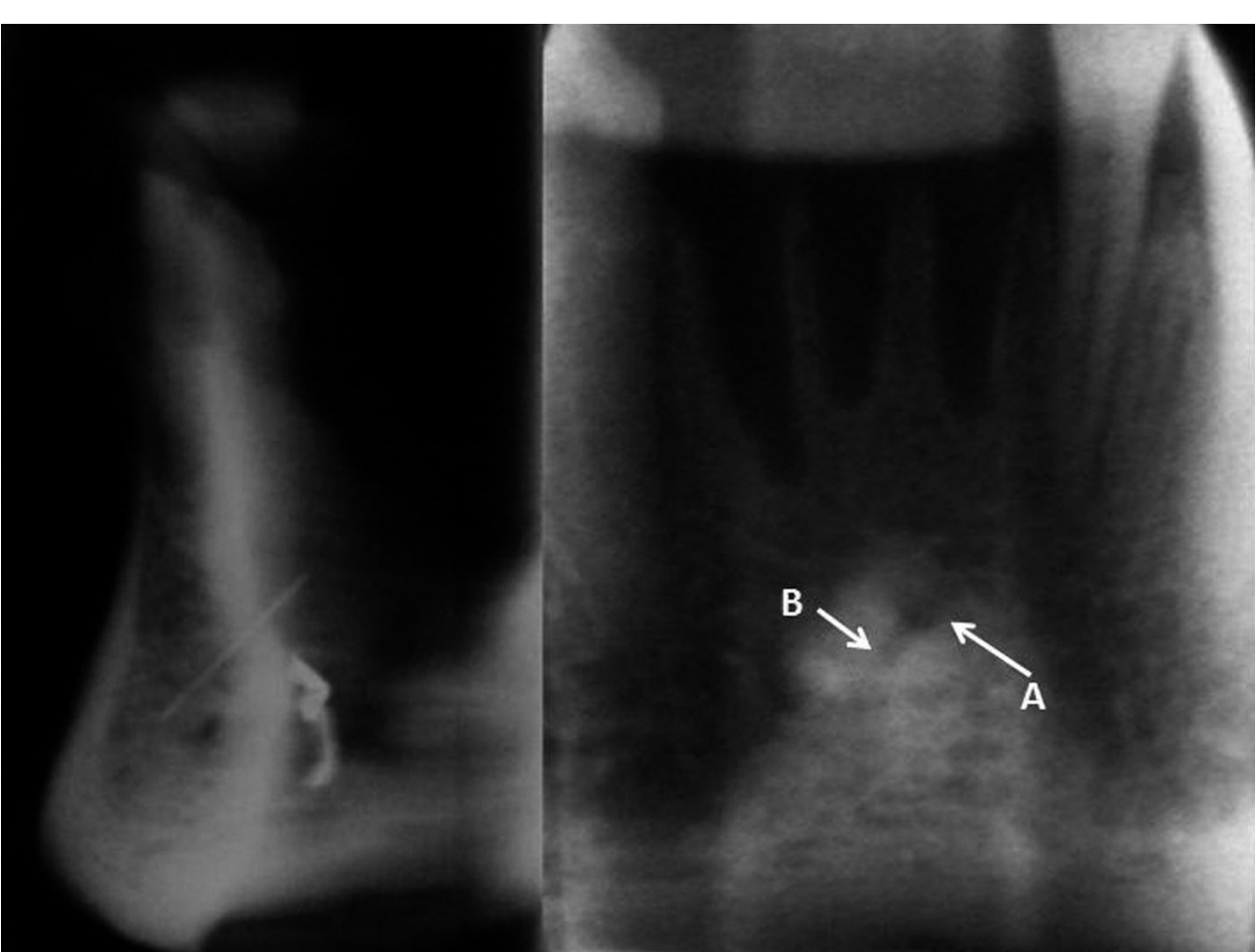

Fig. 3. Imagen izquierda (tomografía lineal) muestra trayectoria ascendente de conducto lingual. En imagen derecha (segmento de radiografía panorámica), se observa tanto el conducto $(A)$ como el foramen lingual $(B)$.

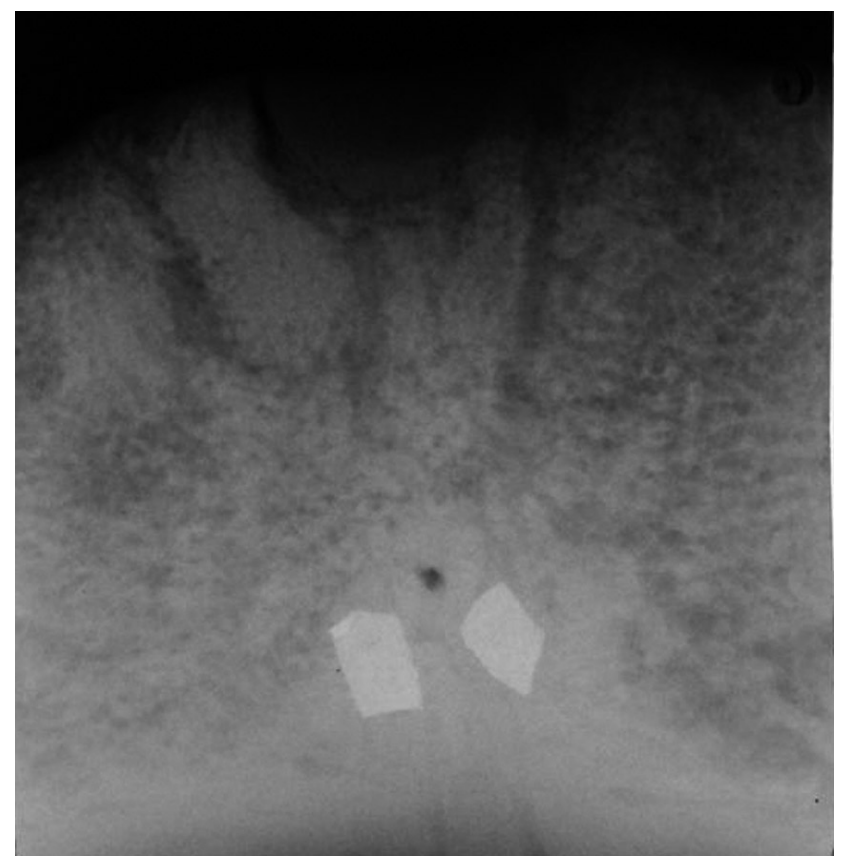

Fig. 2. Espinas mentoniana con marcadores metálicos, sin relación con foramen lingual.

En ocho casos, el conducto lingual produjo la imagen del foramen lingual cuando era ortográfico al paso de los rayos, esto ocurrió tanto en radiografías panorámicas como en periapicales. Sólo en un caso

con panorámicas, y uno con periapicales el foramen lingual no se visualizó, pese a ser ortográfico al paso de los rayos $\mathrm{x}$.

En dos casos en que el conducto lingual no fue ortográfico al paso de los rayos en la radiografía panorámica, el agujero lingual se pudo observar de todas maneras (Fig.3)

Con respecto al número y disposición de conductos linguales, de las 9 mandíbulas, 5 tenían un conducto único, y 4 tenían dos conductos. Cuando se encontró un conducto úni$\mathrm{co}$, siempre tuvo una direc- 


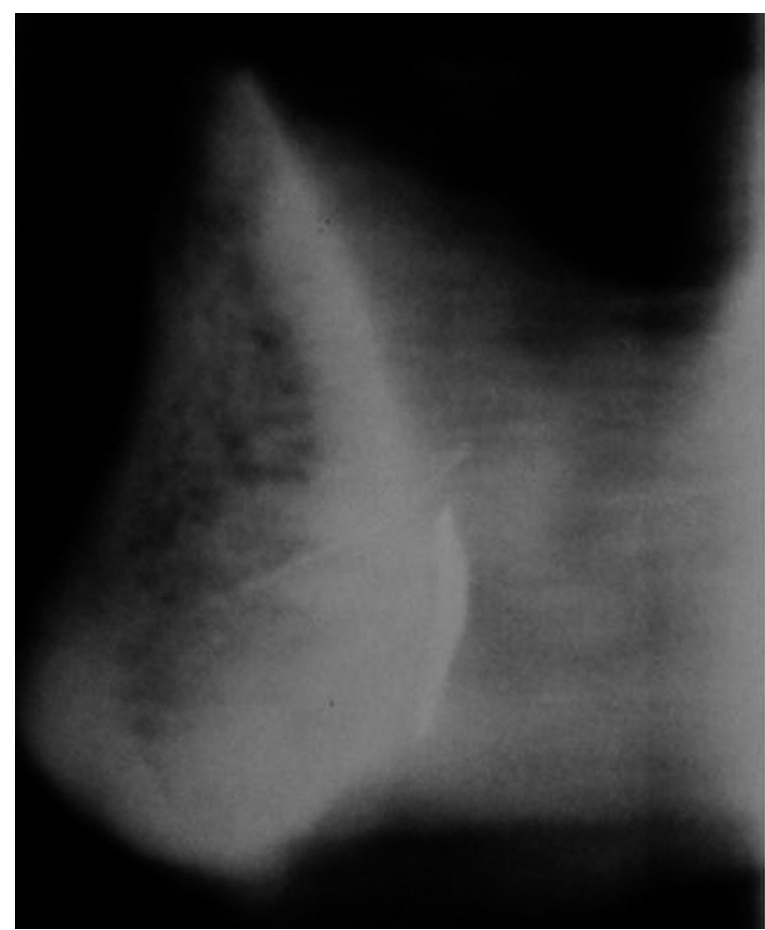

Fig. 4. Se observa conducto lingual con marcador metálico, de trayectoria ascendente, con foramen lingual superior a espinas mentonianas.

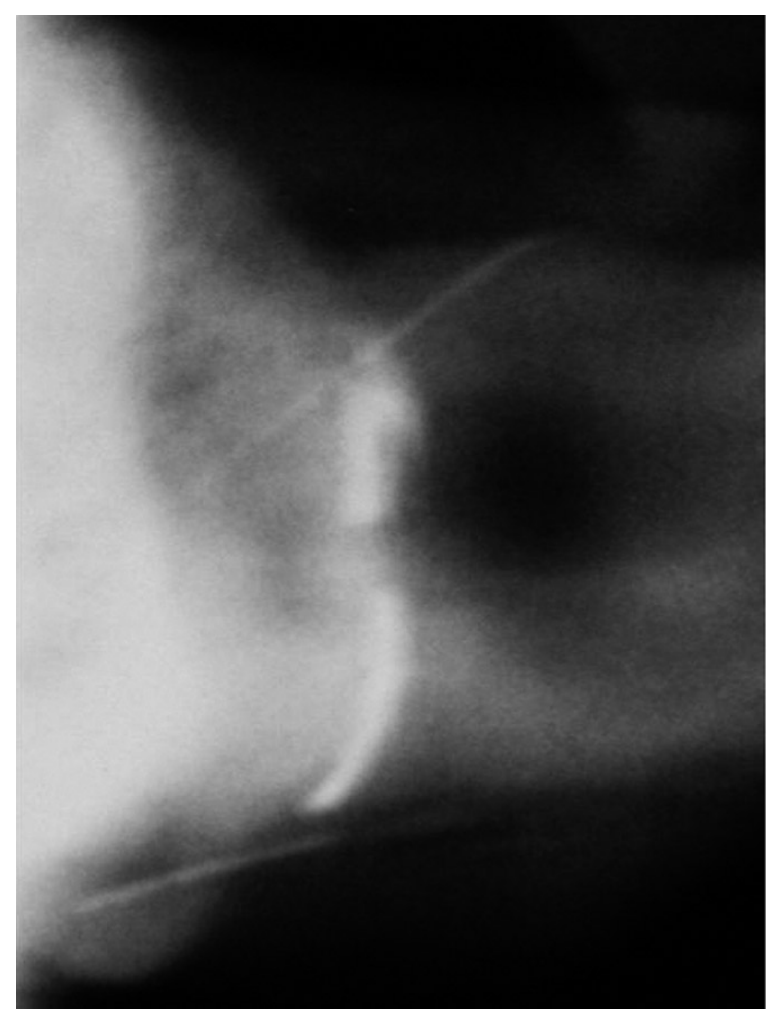

Fig. 5. Se observan dos conductos linguales de trayectoria ascendente, el de arriba, con foramen lingual superior a las espinas mentonianas; el de abajo, con foramen lingual inferior a las espinas mentonianas.

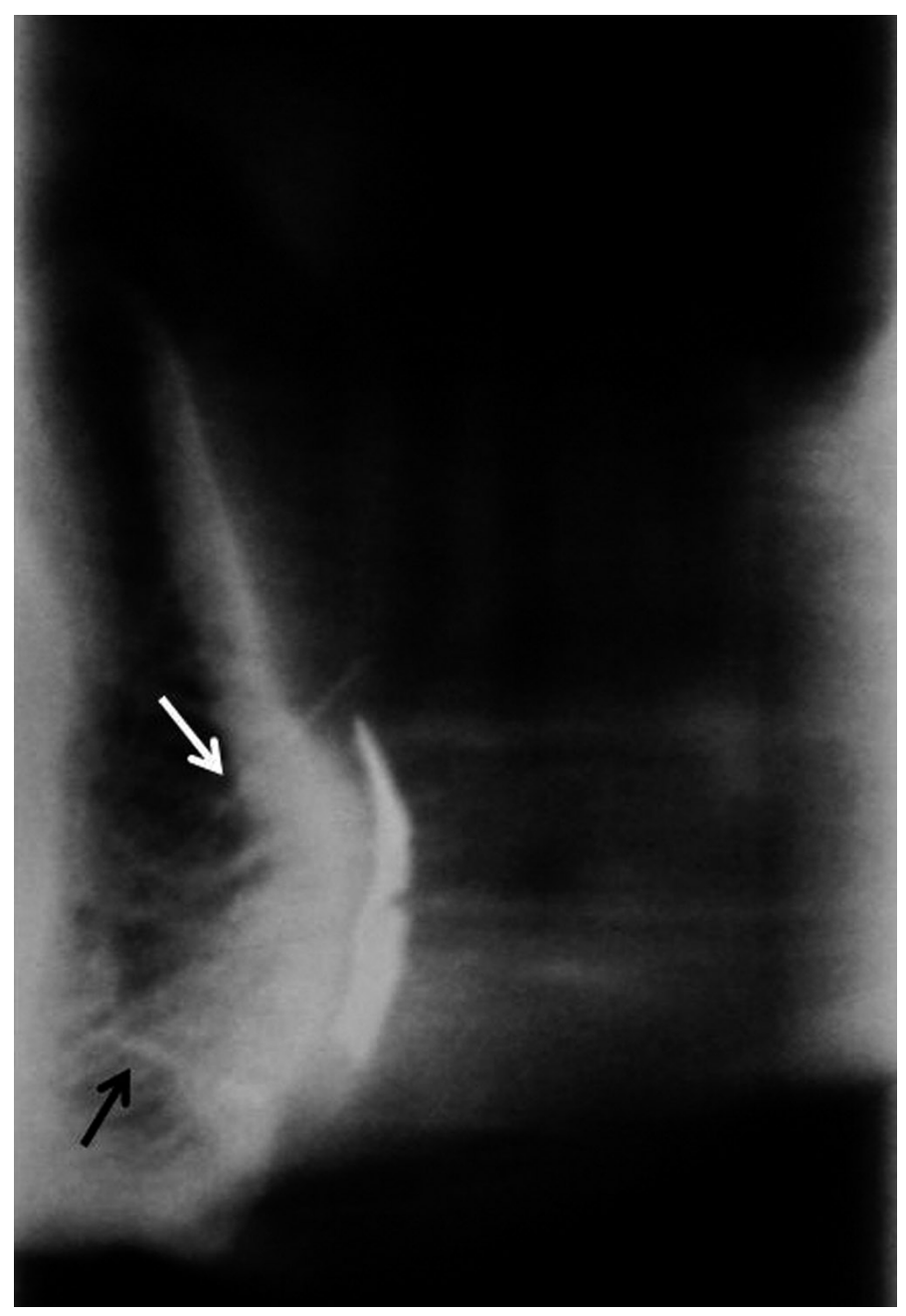

Fig. 6. Se observan dos conductos linguales. Uno de trayectoria ascendente (flecha blanca), con foramen lingual superior a espinas mentonianas, el otro de trayectoria descendente (flecha negra), con formen lingual inferior a espinas mentonianas.

ción ascendente, con su foramen en posición superior (Fig.4). En los 4 casos en que se encontró mandíbulas con dos conductos linguales, dos de ellas tuvieron sus dos conductos ascendentes, con un foramen en posición superior, y el otro en posición inferior (Fig.5), mientras que las otras dos tuvieron un conducto ascendente, con su foramen superior, y un conducto descendente con su foramen inferior (Fig.6).

\section{DISCUSIÓN}

A pesar de que existe controversia respecto del contenido de el o los conductos linguales, (Gomez Mattaldi; Gibilisco; McDonell et al.; Wood \& Goaz; Vasconcellos et al.; Nagar et al.; De Freitas et al.; Liang et al., 2006; Liang 
et al., 2007), existe acuerdo en la posibilidad de complicaciones quirúrgicas en esta zona, tales como el hematoma sublingual (Tepper et al.; Isaacson; Kawai et al., 2007; Rosano et al., 2009). Así, un adecuado conocimiento de las características anatómicas de esta zona y sus variantes debieran ser tomadas en cuenta durante procedimientos quirúrgicos (Tepper et al.; Isaacson; Liang et al., 2007; Rosano et al.).

Varios textos de radiología odontológica describen al agujero lingual rodeado por un área radiopaca producida por las espinas mentonianas (Gibilisco; Haring \& Lind; De Freitas). Así, la descripción clásica del foramen lingual sería en el centro de dichas estructuras. Esto ha llevado a que tradicionalmente en las clases de anatomía radiográfica de la mandíbula se enseñe de esta manera. Esta investigación demostró la no concordancia radiográfica entre las espinas mentonianas y el área radiopaca del foramen lingual, de manera similar a lo señalado por Baldissera \& Silveira y Mraiwa et al. La imagen radiopaca periférica del foramen lingual es explicada por varios autores como la proyección de la cortical del conducto lingual (Goaz \& White; Baldisera \& Silveira; Mraiwa et al.; White \& Pharoa). Del punto de vista absorsoproyeccional, al pasar el rayo de manera ortográfica al conducto, está pasando a su vez ortográfico a sus paredes, lo que produce la imagen radiopaca periférica al foramen que conformaría esta área (McDonnell et al.). Así, el grosor del área radiopaca dependerá del grado de corticalización del conducto lingual. Se ha descrito que la mayoría de los conductos linguales (91\%) tienen un límite bien corticalizado, lo que está significativamente relacionado con su visibilidad en radiografías dentales (Mraiwa et al.). Se debe tener en consideración que cuando no aparece imagen de foramen lingual, no necesariamente significa que no exista dicho foramen, sino puede ser que el rayo no lo haya incidido de forma ortográfica. Una situación no estudiada aún es la posibilidad de que la imagen radiopaca periférica al foramen lingual correspondiera a la corticalización del extremo final del conducto y la corticalización de los bordes del foramen lingual. Esto último podría explicar la aparición radiográfica del foramen lingual, en aquellos casos en que no es ortográfico al paso de los rayos.

En la presente investigación, cuando las eminencias mentonianas estuvieron muy cerca del foramen lingual, éstas se proyectaron, dependiendo de la angulación del rayo, adyacentes a la imagen del foramen, sin embargo, no produjeron la característica área radiopaca.
Con respecto a la frecuencia de aparición del foramen lingual, Baldisera \& Silveira señalan en su estudio que encontraron una presencia del $100 \%$, lo que está cercano a lo encontrado en otros estudios recientes, por lo que les sorprende que esto no esté reflejado en los libros, lo que contribuiría al desconocimiento de los reparos anatómicos estudiados (Liang et al., 2006)

En dos ocasiones fue posible observar el foramen lingual radiográficamente cuando el conducto lingual no estaba ortográfico al paso de los rayos. Del análisis de estos dos casos se desprende que esto es posible cuando el agujero lingual es de mayor tamaño. Lo opuesto ocurrió en dos casos en que el rayo era ortográfico al conducto, pero no se observaba el agujero lingual en las imágenes. Esto podría ocurrir debido al pequeño tamaño de los reparos estudiados (conducto y foramen lingual), y posiblemente a que el conducto lingual no fue ortográfico al rayo en todo su recorrido. Así, es necesario considerar la variable tamaño del foramen lingual y diámetro del conducto lingual, y su incapacidad de producir cambio absorcional. De esta forma adquiere importancia la diferencia de diámetro del conducto lingual, que se ha descrito entre 0,4-1,5 mm. (Gahleitner et al., 2001). Liang et al. (2006) señalaron que es poco probable que conductos linguales de diámetro menor a $1 \mathrm{~mm}$ pudieran causar complicaciones quirúrgicas, en cambio en aquellos sobre $1 \mathrm{~mm}$ debe considerarse esta posibilidad. Del punto de vista radiográfico esta información cobra relevancia ya que podría implicar que la aparición radiográfica en una radiografía periapical del agujero lingual signifique un diámetro mayor a $1 \mathrm{~mm}$. y por lo tanto podría utilizarse como una señal de alerta de riesgo de complicaciones quirúrgicas o postquirúrgicas.

Se ha descrito que personas de mayor edad muestran diámetros menores del conducto lingual de salida inferior (Liang et al., 2006), lo que podría llevar a una menor frecuencia de aparición en radiografías panorámicas, ya que es esta trayectoria de conducto la que es ortográfica al paso de los rayos en esa técnica. Además, esto de alguna manera podría explicar que una mayoría relativa de mandíbulas de este trabajo (5 de 9) tenían sólo un conducto, ya que por ser una muestra de cadáveres posiblemente de edad avanzada, ya no tuvieran su conducto de salida inferior. Con respecto a este tema, se ha reportado que cuando existe un solo foramen, típicamente se encuentra localizado en posición superior (Liang et al., 2007). Esto también podría ser explicado por el hecho de trabajar 
en estudios con mandíbulas de cadáveres, que mayoritariamente son de personas de mayor edad. Esto se contrapone con lo señalado por Mraiwa et al., que encontraron un mayor porcentaje de aparición del foramen lingual en radiografías panorámicas de mandíbulas de cadáveres.

La descripción que hace la literatura sobre la aparición del foramen lingual en radiografías panorámicas, indica que este reparo anatómico aparecería en estos casos cuando el conducto lingual sigue la dirección del rayo. Al contrastar esta información con lo que señalan los estudios en relación con la variación en la trayectoria y número de conductos linguales, sería posible pensar que el agujero lingual que aparece en radiografías periapical y panorámica, podría corresponder a conductos linguales en diferente trayectoria. De lo anterior es posible deducir que cuando se observa un agujero lingual en radiografías periapical y panorámica del mismo paciente, esto podría indicar la presencia de dos conductos linguales. Así, el foramen que aparece en la radiografía periapical sería ortográfico al paso de los rayos $X$, teniendo una inclinación ascendente, mientras que el foramen que se observa en la radiografía panorámica podría corresponder a un conducto lingual de orientación descendente (Fig.7). Sin embargo este análisis no sería concluyente, ya que se debe considerar la variable diámetro señalada anteriormente, y la posibilidad de que una mayor corticalización de los bordes del foramen lingual tam- bién tengan un efecto en la aparición de este reparo en la radiografía.

Se ha descrito que en imágenes de tomografía espiral de la línea media mandibular la visualización del foramen lingual y el conducto lingual es superior en comparación con estudios con radiografías convencionales. Esto último podría ser atribuido a las ventajas inherentes del la imágenes óseas de cortes transversales, ya que la visibilidad en otras imágenes bidimiensionales (intraorales, panorámica), está limitada y depende de la proyección geométrica, además de otros factores tales como el grado de corticalización de las paredes del canal (Mraiwa et al.).

Se ha señalado que la frecuencia de aparición del foramen lingual en radiografías panorámicas es mayor que en retroalveolares (Mraiwa et al.), debido a que la trayectoria del conducto lingual es generalmente ortográfica al paso de los rayos $\mathrm{X}$. Esto no concuerda el trabajo de Liang et al., (2006) en imágenes de scanner helicoidal de 555 mandíbulas, quienes encontraron un $53 \%$ de forámenes linguales superiores(con un $45 \%$ de trayectoria ascendente y un $8 \%$ de trayectoria horizontal) y $47 \%$ inferiores a las espinas mentonianas, todos de trayectoria descendente. Estos estudios hacen pensar que la mayor aparición del foramen lingual en radiografías panorámicas se deba a la aparición en éstas tanto de aquellos forámenes de trayectoria ascendente como horizontal.
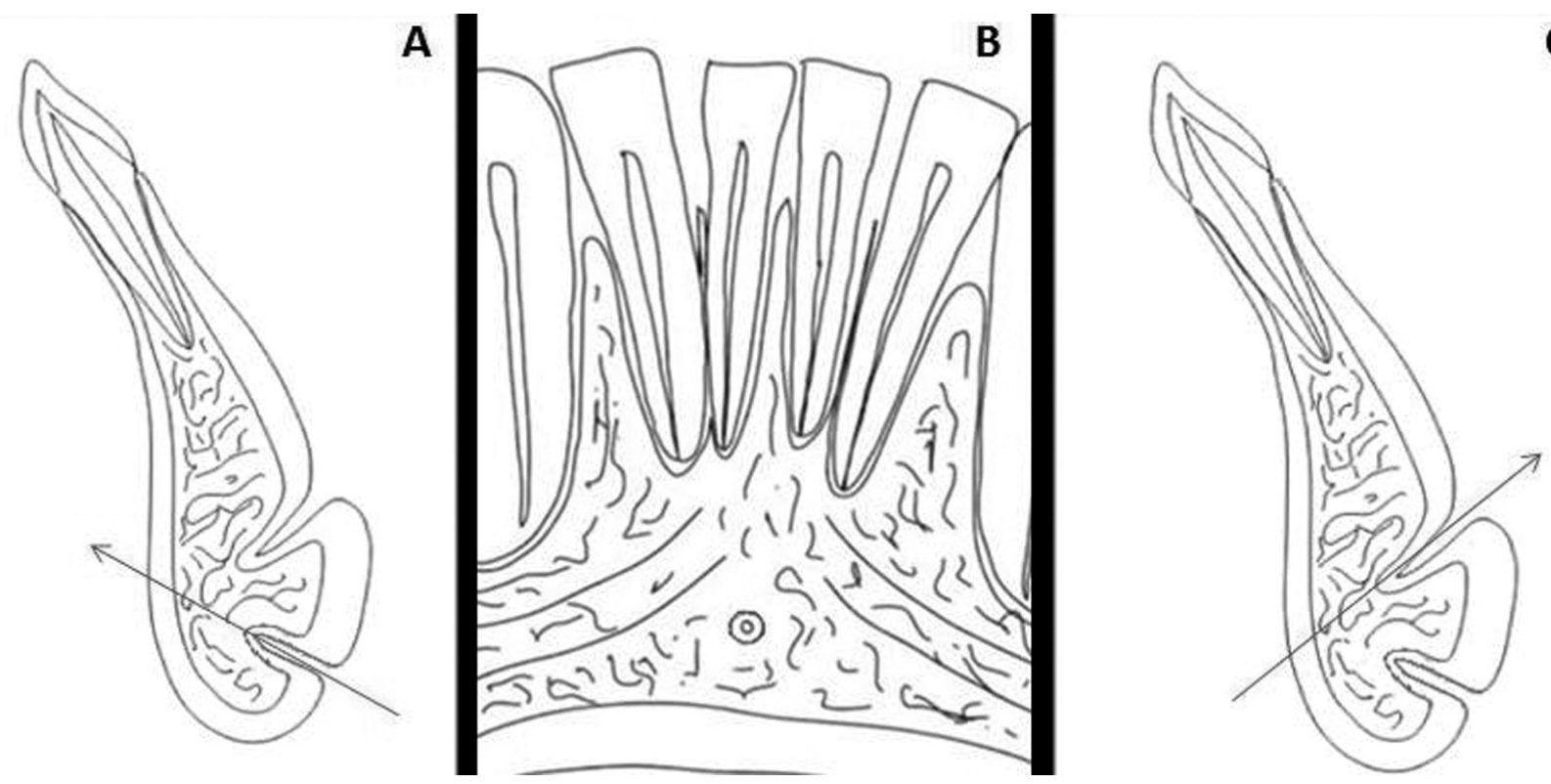

Fig. 7. Imagen A grafica el paso del rayo X (flecha roja) en una radiografía panorámica. Imagen $\mathrm{C}$ grafica el paso del rayo $\mathrm{X}$ (flecha roja) en una radiografía periapical. Imagen B muestra el resultado radiográfico de cualquiera de los dos casos. 
Del punto de vista absorso-proyeccional, cuando el conducto lingual no es incidido de manera ortográfica por los rayos $X$, sino en superficie, podría ser observado en las radiografías como una cinta radiolúcida limitada por delgadas líneas radiopacas correspondiente a las corticales laterales del conducto lingual. En el presente trabajo sólo se pudo observar esta situación en dos casos, en ninguno de los dos se observó un límite corticalizado, sino más bien un límite definido (Fig.3 B). En el resto de los casos, el conducto sólo fue distinguible gracias el marcador metálico en su interior.

La literatura señala la presencia de canales linguales bifurcados (Vasconcellos et al.; Liang et al., 2006). La metodología utilizada en el presente estudio no permitió analizar esta variable. Sería interesante estudiar esta materia con otras medios imagenológicos disponibles tales como el cone beam CT.

Liang et al. (2006) encontraron efectos entre el sexo y edad con respecto a características dimensionales del conducto lingual, encontrando un diámetro menor, posición más baja, y que las mujeres tenían conductos linguales tanto superiores como in- feriores de mayor diámetro que los hombres. Ellos también encontraron una mayor tendencia de los hombres a tener dos conductos linguales y de las mujeres, a tener un solo conducto lingual. Sería interesante estudiar el conducto lingual en distintos grupos etarios y por sexo para caracterizar de mejor forma este reparo anatómico en la población chilena. El cada vez mejor acceso a estudios con tomografía computarizada permitiría realizar un estudio en esta línea.

Del punto de vista clínico, es importante señalar que la aparición del foramen lingual en radiografías periapicales es un indicador de que el conducto lingual tiene una trayectoria ascendente, y además tiene un calibre no menor que le permite aparecer en la imagen radiográfica, por lo que sería un indicador de riesgo mayor de daño potencial durante un acto quirúrgico en la zona. Esto es de alguna manera planteado por Liang et al. (2007), quienes señalaron que sólo aquellos pacientes con un conducto único inferior no tienen riesgo de daño del canal durante una cirugía de la región. Lo señalado podría indicar que la aparición del foramen lingual en una radiografía panorámica advertiría la presencia de un conducto lingual de trayectoria descendente, por lo tanto de bajo riesgo de daño quirúrgico.

HIDALGO, A. \& SAN PEDRO, J. Relationship between lingual foramen and mental spines, and evaluation of lingual canal by using intra and extra-oral radiographs. Int. J. Odontostomat., 4(3):295-302, 2010.

ABSTRACT: Radiological location of lingual foramen is described by traditional dental literature in the median line in the lingual aspect of the mandible, between mental spines, both in periapical and panoramic radiographs. Traditionally the radiopaque area surrounding the lingual foramen is described as produced by mental spines. This interpretation currently is controversial. The aim of this study was to determine the relationship of lingual foramen with mental spines by looking directly at the dry mandibles, and by looking at periapical and panoramic radiographs; to determine trajectory of lingual canal by using linear tomographies of the area of interest, and describe radiographic representation from the absorption projectional point of view. This investigation demonstrated that the radiopaque area surrounding the lingual foramen is not produced by mental spines.

KEY WORDS: dental, radiography, mandible, median line, anatomical landmarks, panoramic, radiography, mental spines, lingual foramen.

\section{REFERENCIAS BIBLIOGRÁFICAS}

Baldissera, E. Z. \& Silveira, H.D. Radiographic evaluation of the relationship between the projection of genial tubercles and the lingual foramen. Dentomaxillofacial Radiology, 31:368-72, 2002.

Basrani, E.; Blank, A. J.; Cañete, M.T. Radiología en Endodoncia. Buenos Aires. Actualidades Médico Odontológicas Latinoamérica C. A., 2002.

De Freitas, A.; Rosa, J.E. \& Faría e Souza, I. Radiolo- gía Odontológica. São Paulo, Editora Artes Médicas Ltda., 2002.

Figún, M. E.; Garino, R.R. Anatomía Odontológica Funcional Aplicada. $2^{\text {a }}$ Ed. Buenos Aires, El Ateneo, 2002.

Gahleinter, A.; Hofschneider, U.; Tepper, G.; Pretterklieber, M.; Schick, S.; Zauza, K. \& Watzek, G. Lingual Vascular Canals of Mandible: Evaluation with Dental CT. Radiology, 220:186-9, 2001. 
HIDALGO, A. \& SAN PEDRO, J. Relación del foramen lingual con espinas mentonianas y evaluación del conducto lingual mediante radiografías intra y extraorales. Int. J. Odontostomat., 4(3):295-302, 2010

Gibilisco, J.A. Diagnóstico Radiológico en Odontolo-

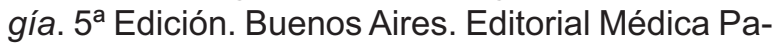
namericana S. A., 1994.

Goaz, P.W. \& White, S.C. Radiología Oral. Principios e Interpretación. $3^{a}$ Ed. Madrid. Editorial Mosby/ Doyma Libros. 1995.

Gomez Mattaldi, R.A. Radiología Odontológica. Buenos Aires. Editorial Mundi S.A.I.y F., 1979.

Haring, J.I; Lind, L.J. Radiología Dental. Principios y Técnicas. México, D.F. McGraw-Hill Interamericana Editores, 1997.

Isaacson, T.J. Sublingual hematoma formation during immediate placement of mandibular endosseous implants. J. Am. Dent. Assoc., 135:168-72, 2004.

Kwai, T.; Asaumi, R.; Sato, I.; Yoshida Sh.; Yosue, T. Classification of the lingual foramina and their bony canals in the median region of the mandible: cone beam computed tomography observations of dry Japanese mandibles. Oral Radiol., 23:42-8, 2007.

Langlais, R. P; Kasle M. J. Excercises in oral radiographic interpretation. Third ed. Philadelphia. W.B. Saunders Company, 1992.

Liang, X.; Jacobs R. \& Lambrichts I. An assessment on spiral CT scan of the superior and inferior genial spinal foramina and canals. Surg. Radiol. Anat., 28: 98-104, 2006.

Liang, X; Jacobs, R; Lambritchs, Vanderwalle, G. Lingual foramina on the mandibular midline revisited: A Macroanatomical study. Clinical Anatomy, 20:246-251, 2007.

McDonnel, D.; Reza Nouri, M. \& Todd, M.E. The mandibular lingual foramen: a consistent arterial foramen in the middle of the mandible. J. Anat., 184: 363-369, 1994.

Mraiwa, N.; Jacobs, R.; Moerman, P.; Lambrichts, I.; van Steenberghe, D. \& Quirynen, M. Presence and course of the incisive canal in the human mandibular interforaminal region: two-dimensional imaging versus anatomical observations. Surg. Radiol. Anat., 416 -423, 2003.

Nagar, M.; Bhardwaj, R. \& Prakash, R. Accessory Lingual Foramen in Adult Indian Mandibles. J. Anat.
Soc. India. 50(1):13-4, 2001.

Rosano, G.; Taschieri, S.; Gaudy, J.F.; Testori, T. \& Del Fabbro, M. Anatomic Assesment of the anterior mandible and relative hemorrage risk in implant dentistry: a cadaveric study. Clin. Oral Impl. Res. 20:791-5, 2009.

Rouvière H,; Delmas, A. Anatomía Humana Descriptiva, Topográfica y Funcional. Tomo I. Cabeza y CueIlo. 10ª edición. Barcelona. Editorial Masson, 1999.

Tepper, G.; Hofschneider, U.B.; Gahleitner, A. \& Ulm, C. Computed tomographic diagnosis and localization of bone canals in the mandibular interforaminal region for prevention of bleeding complications during implant surgery. Int. J. Oral Maxillofac. Implants, 16:68-72, 2001.

Vasconcellos, H. A.; Siquerira, E. A.; Almeyda, G. H.; Tan, M. L. \& Barros, P. H. The anatomy of the lingual foramen canal and its related to the mandibular symphysis. Rev. Chil. Anat., 18(1):47-51, 2000.

Velayos, J. L. \& Santana H. Anatomía de la Cabeza con Enfoque Odontoestomatológico. $2^{\mathrm{a}}$ edición. Madrid. Editorial Médica Panamericana, 1998.

White, S.C. \& Pharoa, M.J. Oral Radiology. Principles and Interpretation. Fifth Ed. St. Louis, Mosby, 2004.

Wood, N.K. \& Goaz, P.W. Diagnóstico Diferencial de las Lesiones Orales y Maxilofaciales. Barcelona, Harcourt Brace de España, S.A., 1999.

Dirección para correspondencia:

Alejandro Hidalgo Rivas

Servicio de Radiología

Departamento de Estomatología

Facultad de Ciencias de la Salud

Universidad de Talca

Avenida Lircay s/n

Talca

CHILE

Tel.: 56-71-200476

Email: ahidalgo@utalca.cl

Recibido : 15-10-2010

Aceptado: 18-11-2010 